\title{
Detector de bordas com maior precisão de localização e robustez a variações de brilho e contraste da imagem
}

\author{
Marcelo Perez e Carla Pagliari
}

\begin{abstract}
Resumo - Este artigo apresenta um detector de bordas com maior precisão de localização e robustez a variações de brilho e contraste da imagem. Após um preciso desenvolvimento da equação diferencial a ser satisfeita pela intensidade da imagem em pontos de borda, esta condição é verificada para cada pixel utilizando-se cruzamento por zeros de um operador definido sobre um espaço de escalas. Utilizando-se verificações adicionais para a seleção de candidatos a pontos de contorno, o algoritmo fornece bordas finas e bem localizadas, minimizando a ocorrência de falsos positivos e negativos, e apresenta resultados superiores quando comparado a diversos detectores de borda de referência.
\end{abstract}

Palavras-chave - detecção de bordas, extração de contornos.

Abstract - This paper presents an edge detector that accurately localizes edges yet remaining robust to brightness and contrast variation in images. After the precise development of the differential equation that must be satisfied by the image intensity at edge points, this condition is verified for every pixel using zero-crossing of a space scale operator. Moreover, by applying checking schemes to the selection of edge candidate points, false responses are minimized, and thin edges are provided. The method showed superior results when compared with well-known edge detectors.

Keywords - edge detection, edge extraction.

\section{INTRODUÇÃOO}

O filtro gaussiano apresenta algumas propriedades importantes, que o tornaram uma das ferramentas de filtragem mais utilizadas em processamento de imagens. Em detecção de bordas, tem-se utilizado este filtro desde que Marr e Hildreth [1] demonstraram que o filtro gaussiano, em conjunto com o operador laplaciano, é uma aproximação da forma dos campos receptores espaciais do sistema visual dos gatos (o que também foi proposto para o sistema visual humano). Através de métodos variacionais, Canny [2] propôs um detector de bordas ótimo que pode ser aproximado pela primeira derivada de uma função gaussiana. Juntamente com o laplaciano, a função gaussiana é o único filtro que não cria novos cruzamentos por zeros com o aumento de escala. O filtro gaussiano bi-dimensional (2-D) é o único filtro rotacionalmente simétrico que é separável em coordenadas cartesianas.

Marr e Hildreth [1] introduziram formalmente o filtro gaussiano no processo de detecção de bordas baseados no estudo da visão biológica, ao utilizar o operador laplaciano da gaussiana (LoG) como um filtro. No entanto, em seu trabalho, Marr e Hildreth aproximaram a segunda derivada de uma função 2-D pelo laplaciano, que é uma função escalar, enquanto as primeiras derivadas de uma função 2-D definem campos de vetores. Além disto, um ponto de borda pode ser definido como um ponto no qual a magnitude do gradiente assume um máximo ao longo da própria direção do gradiente [2]. Isto implica que, rigorosamente, é

Marcelo Perez, Carla Pagliari, Departamento de Engenharia Elétrica, Instituto Militar de Engenharia, Rio de Janeiro, Brasil.

E-mails: perez@ime.eb.br, carla@ime.eb.br necessário o cálculo da derivada direcional no campo do gradiente para que se possa corretamente obter um ponto de borda.

Embora muitos trabalhos apresentando detectores de bordas tenham sido publicados $[4,5]$, este artigo propõe uma implementação mais exata da abordagem original de Marr e Hildreth. As principais modificações são o desenvolvimento de uma formulação matemática mais precisa para pontos de borda e a determinação destes pontos através de cruzamentos por zero da segunda derivada, calculada de forma direcional sobre a direção do vetor gradiente (pois imagens são funções 2-D). A convolução da imagem com um espaço de escalas de filtros gaussianos é mantida para redução dos efeitos do ruído sobre as operações de derivação e para a própria implementação numérica das diferenciações. A motivação para o desenvolvimento deste detector de bordas advém do fato de que um extrator de contornos com uma resposta tão invariante quanto possível a mudanças de iluminação da cena é uma característica extremamente desejável. Isto é especialmente interessante para aplicações em sistemas automatizados, que não requeiram a interferência de um operador humano. Além disso, um detector de bordas que produza bordas finas, com precisão de localização e que seja imune a variações de brilho e contraste pode ser importante não somente em sistemas automatizados, mas também em outras aplicações que necessitem elevado desempenho.

Este artigo está organizado da seguinte forma: a Seção II apresenta o método proposto, a Seção III mostra os resultados obtidos e os compara com os produzidos por alguns dos principais detectores de borda em uso como o de Canny [2], Marr-Hildreth [1] e SUSAN [3]; finalmente, na Seção IV, são apresentadas as conclusões.

\section{O DETECTOR DE BORDAS}

Neste trabalho, consideramos que a abordagem introduzida por Marr e Hildreth [1] é válida, no sentido de que uma borda é detectada através do cruzamento por zero da segunda derivada. Eventuais falhas ocorrem primordialmente devido a quatro fatores, a saber:

- a segunda derivada não é calculada ao longo da direção do gradiente;

- $\quad$ esta segunda derivada direcional é aproximada pelo laplaciano (um operador escalar);

não é verificado se este cruzamento por zero corresponde a um máximo, mínimo ou ponto de inflexão;

não existe qualquer processo de afinamento de bordas.

Com o objetivo de se deduzir as expressões matemáticas que definem o detector de bordas proposto, é importante ressaltar que a projeção do gradiente sobre um vetor unitário (o produto escalar) é a taxa de variação da função 2-D na direção deste vetor unitário. Assim sendo, um ponto de borda pode ser definido como um ponto em que a magnitude do gradiente assume um máximo local na direção do gradiente [2]. As expressões matemáticas apresentadas a seguir estão de acordo com a situação ilustrada na Figura 1. 


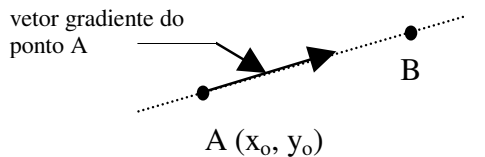

Fig. 1. O ponto B está a $|\Delta|$ unidades do ponto de borda A.

Suponhamos que A seja um ponto de borda e que B seja outro ponto, situado ao longo da direção do gradiente de A. Suponhamos também que $\mathrm{B}$ se encontra unidades $|\Delta|$ de comprimento distante de A (Fig 1), onde $\Delta$ é muito pequeno. A rigor, o gradiente no ponto B não possui a mesma direção que o vetor gradiente no ponto A. A projeção do gradiente em $\mathrm{B}$ sobre o vetor unitário com a mesma direção do gradiente em A é dada por:

$$
\begin{aligned}
\mathrm{P}(\Delta)= & \frac{1}{\mathrm{~K}} \frac{\partial \mathrm{I}\left(\mathrm{x}_{0}, \mathrm{y}_{0}\right)}{\partial \mathrm{x}} \frac{\partial \mathrm{I}\left(\mathrm{x}_{0}+\frac{\partial \mathrm{I}\left(\mathrm{x}_{0}, \mathrm{y}_{0}\right)}{\partial \mathrm{x}} \Delta, \mathrm{y}_{0}+\frac{\partial \mathrm{I}\left(\mathrm{x}_{0}, \mathrm{y}_{0}\right)}{\partial \mathrm{y}} \Delta\right)}{\partial \mathrm{x}}+(1) \\
& \frac{1}{\mathrm{~K}} \frac{\partial \mathrm{I}\left(\mathrm{x}_{0}, \mathrm{y}_{0}\right)}{\partial \mathrm{y}} \frac{\partial \mathrm{I}\left(\mathrm{x}_{0}+\frac{\partial \mathrm{I}\left(\mathrm{x}_{0}, \mathrm{y}_{0}\right)}{\partial \mathrm{x}} \Delta, \mathrm{y}_{0}+\frac{\partial \mathrm{I}\left(\mathrm{x}_{0}, \mathrm{y}_{0}\right)}{\partial \mathrm{y}} \Delta\right)}{\partial \mathrm{y}}
\end{aligned}
$$

, onde K é a magnitude do vetor gradiente no ponto A, dada por:

$K=\sqrt{\left(\frac{\partial \mathrm{I}\left(\mathrm{x}_{\mathrm{o}}, \mathrm{y}_{\mathrm{o}}\right)}{\partial \mathrm{x}}\right)^{2}+\left(\frac{\partial \mathrm{I}\left(\mathrm{x}_{\mathrm{o}}, \mathrm{y}_{\mathrm{o}}\right)}{\partial \mathrm{y}}\right)^{2}}$

$\mathrm{P}(\Delta)$ é uma função real que associa a cada escalar $\Delta$ a projeção do gradiente no ponto $\mathrm{B}=\left(\mathrm{x}_{\mathrm{o}}+\frac{\partial \mathrm{I}\left(\mathrm{x}_{\mathrm{o}}, \mathrm{y}_{\mathrm{o}}\right)}{\partial \mathrm{x}} \Delta, \mathrm{y}_{\mathrm{o}}+\frac{\partial \mathrm{I}\left(\mathrm{x}_{\mathrm{o}}, \mathrm{y}_{\mathrm{o}}\right)}{\partial \mathrm{y}} \Delta\right)$ sobre o vetor unitário com a mesma direção do vetor gradiente no ponto A.

Se o ponto A for um ponto de borda, e de acordo com a definição aqui adotada para um ponto de borda, a função $\mathrm{P}(\Delta)$ deverá exibir um máximo local para $\Delta=0$. Supondo que a função $\mathrm{P}(\Delta)$ seja contínua e que a sua primeira derivada seja definida para $\Delta=0$, então o fato de que A é um ponto de borda implica que a primeira derivada $\mathrm{P}^{\prime}(\Delta)$ de $\mathrm{P}(\Delta)$ deverá ser zero para $\Delta=0$.

Como, para um ponto de borda, o vetor gradiente é um vetor não nulo, sua magnitude K dada pela Equação (2) não pode ser igual a zero. Além disto, iremos supor que a função intensidade da imagem apresente segundas derivadas contínuas (e neste caso a ordem em que as derivadas de segunda ordem são computadas pode ser permutada); então, a partir da Equação (1), chega-se à Equação (3). Assim, a condição necessária para que um ponto seja considerado pertencente a uma borda é que, para este ponto, a seguinte condição seja satisfeita:

$$
\left(I_{x}\right)^{2} I_{x x}+2 I_{x} I_{y} I_{x y}+\left(I_{y}\right)^{2} I_{y y}=0
$$

onde $I_{x}$ e $I_{y}$, são os valores das primeiras derivadas parciais da intensidade dos pixels ao longo das direções $\mathrm{x}$ e y respectivamente, $\mathrm{e} \mathrm{I}_{\mathrm{xx}}$, $I_{y y}$ e $I_{x y}$ são os valores das segundas derivadas parciais da intensidade dos pixels ao longo da direção $\mathrm{x}$, ao longo da direção y e ao longo das direções x e y, respectivamente.

A Equação (3) atende à condição de que a segunda derivada ao longo da direção do gradiente deva ser igual a zero para que o ponto seja considerado um ponto de borda. Outra condição importante é que o ponto deve corresponder a um máximo da derivada de primeira ordem na direção do gradiente. Esta última condição deve-se ao fato de que a Equação (3) também é satisfeita se o ponto da imagem considerado apresenta um mínimo ou um ponto de inflexão para a sua primeira derivada ao longo da direção de seu gradiente. Para garantir a precisão do método, esta condição de máximo deve ser verificada. Matematicamente, isto corresponde à condição de que a terceira derivada ao longo da direção do gradiente deve ser negativa para o ponto considerado. No entanto, tal verificação não só iria impor uma sobrecarga computacional ao algoritmo, mas acabaria levando à inclusão de derivadas de ordem mais elevada, que são altamente sensíveis ao ruído. Todavia, esta condição também necessita ser testada, e será conduzida diretamente sobre os módulos dos vetores gradiente dos pontos candidatos a borda (passo 5 do método apresentado na próxima página).

No cálculo das derivadas parciais da Equação (3), repetimos o procedimento, apresentado em [1], de convolução da imagem com o filtro gaussiano. Devido à sua característica passa-baixas, o filtro gaussiano reduz alguns efeitos do ruído causados pela operação de diferenciação. O comportamento da convolução de duas funções com respeito à diferenciação torna a convolução com a gaussiana muito importante para a implementação da operação de diferenciação propriamente dita. Isto é uma consequência da propriedade da diferenciação da operação de convolução, apresentada na Equação (4), onde $\mathrm{x}(\mathrm{t}) \mathrm{e}$ h(t) são funções arbitrárias.

$\frac{\mathrm{d}}{\mathrm{dt}}[\mathrm{h}(\mathrm{t}) * \mathrm{x}(\mathrm{t})]=\frac{\mathrm{d}}{\mathrm{dt}}[\mathrm{h}(\mathrm{t})] * \mathrm{x}(\mathrm{t})=\mathrm{h}(\mathrm{t}) * \frac{\mathrm{d}}{\mathrm{dt}}[\mathrm{x}(\mathrm{t})]$

Devido à propriedade apresentada pela Equação (4), as derivadas parciais da Equação (3) não precisam ser computadas diretamente sobre as intensidades dos pixels. Desta forma, o filtro gaussiano também tem um papel ativo na implementação numérica do método.

Denotando por $\mathrm{G}(\mathrm{x}, \mathrm{y})$ o filtro gaussiano aplicado à imagem, temse:

$\mathrm{G}(\mathrm{x}, \mathrm{y})=\frac{1}{\sqrt{2 \pi \sigma}} \mathrm{e}^{-\frac{\mathrm{x}^{2}+\mathrm{y}^{2}}{2 \sigma^{2}}}$

Então, se as derivadas parciais da Equação (3), em vez de serem aplicadas diretamente sobre a função intensidade da imagem, forem computadas na imagem filtrada pelo filtro gaussiano, a Equação (3) pode ser escrita como:

$\left(\mathrm{G}_{\mathrm{x}} * \mathrm{I}\right)^{2}\left(\mathrm{G}_{\mathrm{xx}} * \mathrm{I}\right)+2\left(\mathrm{G}_{\mathrm{x}} * \mathrm{I}\right)\left(\mathrm{G}_{\mathrm{y}} * \mathrm{I}\right)\left(\mathrm{G}_{\mathrm{xy}} * \mathrm{I}\right)+\left(\mathrm{G}_{\mathrm{y}} * \mathrm{I}\right)^{2}\left(\mathrm{G}_{\mathrm{yy}} * \mathrm{I}\right)=0$

, onde $\mathrm{G}_{\mathrm{x}}$ e $\mathrm{G}_{\mathrm{y}}$ são os valores das derivadas parciais do filtro gaussiano ao longo das direções x e y respectivamente, e $G_{x x}, G_{y y}$ e $G_{x y}$ são as segundas derivadas parciais do filtro gaussiano ao longo da direção $\mathrm{x}$, ao longo da direção y e ao longo das direções x e y respectivamente.

No entanto, cada convolução dentro dos parênteses da Equação (6) possui implementação rápida, uma vez que as derivadas parciais da função gaussiana, presentes na Equação (6), são separáveis em suas duas variáveis. Consequentemente, as convoluções 2-D da Equação (6) podem ser decompostas como duas convoluções 1-D independentes, computadas ao longo das linhas e das colunas da imagem. Igualmente importante sob o aspecto computacional é o fato do cômputo das Equações (6) e (7) (esta última a ser apresentada a seguir) ser 
paralelizável, uma vez que seus termos podem ser calculados independentemente.

Após este equacionamento, podemos implementar o método proposto de acordo com os seguintes passos:

- 1: Para cada pixel, calcula-se a função S(x,y) dada pela Equação (7):

$\mathrm{S}(\mathrm{x}, \mathrm{y})=\left(\mathrm{G}_{\mathrm{x}} * \mathrm{I}\right)^{2}\left(\mathrm{G}_{\mathrm{xx}} * \mathrm{I}\right)+2\left(\mathrm{G}_{\mathrm{x}} * \mathrm{I}\right)\left(\mathrm{G}_{\mathrm{y}} * \mathrm{I}\right)\left(\mathrm{G}_{\mathrm{xy}} * \mathrm{I}\right)+\left(\mathrm{G}_{\mathrm{y}} * \mathrm{I}\right)^{2}\left(\mathrm{G}_{\mathrm{yy}} * \mathrm{I}\right)(7)$

- 2: Para cada pixel da imagem, verifica-se se ocorre um cruzamento por zero da função $\mathrm{S}(\mathrm{x}, \mathrm{y})$ em uma das quatro direções principais (horizontal, vertical e as duas diagonais). Se não existir cruzamento, este pixel não é um candidato a borda.

- 3: Se o pixel apresenta um cruzamento por zero, deve-se testar se este cruzamento por zero ocorre na direção do gradiente daquele pixel. Para isso, estima-se a direção do gradiente, uma vez que os valores de $\left(G_{x}{ }^{*}\right)$ e $\left(G_{y}{ }^{*} I\right)$ já foram obtidos no cômputo da Equação (7).

- 4: Como uma estimativa da direção do gradiente foi obtida no passo 3 , verifica-se se o cruzamento por zero da função $S(x, y)$ ocorreu nesta direção (dada pelo passo 3) do gradiente. Este é um outro pré-requisito para que um pixel seja considerado um ponto de borda.

- 5: Se o pixel passou no teste do passo 4, verifica-se se a magnitude do vetor gradiente para este pixel é um máximo local na direção obtida no passo 3.

- 6: Se o pixel passou no teste do passo 5, o pixel é considerado um ponto de borda.

Um ponto importante a ser ressaltado é que, dado que o passo 3 especifica a direção do vetor gradiente em um ponto de borda , a direção da borda também é fornecida pelo método proposto.

\section{RESULTADOS}

Nesta seção apresentamos os mapas de borda obtidos pelos métodos de Marr-Hildreth [1], Canny [2], SUSAN [3] e pelo detector de contornos proposto neste artigo. $\mathrm{O}$ objetivo básico é comparar não apenas a precisão de localização dos diferentes métodos, mas também sua invariância a manipulações de brilho e contraste nas imagens. Aqui, a idéia básica subjacente é que um detector deve ser capaz de detectar bordas (geralmente modeladas como degraus) independentemente de suas amplitudes, simultaneamente minimizando o número de falsos positivos e negativos.

Foram utilizadas nas simulações as imagens de teste empregadas em [3], juntamente com imagens disponibilizadas em [6] e outras imagens selecionadas pelos autores. Estas imagens compreendem diferentes graus de detalhamento e textura, de forma a melhor analisar o desempenho dos detectores.

A fim de se verificar a invariância dos detectores de borda a variações de brilho e contraste das imagens, os algoritmos testados foram aplicados às imagens de teste em suas versões originais e 'modificadas'. Estas versões 'modificadas' foram criadas pela aplicação de transformações lineares (isto é, multiplicação por um fator de escala fixo seguido de translação do nível médio) e não-lineares sobre as intensidades destas imagens. Um exemplo de transformação não-linear utilizada é mostrado na Figura 2, na qual os eixos horizontal e vertical representam os níveis de cinza das intensidades (no intervalo 0 a 255) para as imagens original e 'modificada', respectivamente. No contexto deste trabalho, esta transformação mostrada na Figura 2 foi denominada 'Transformação Não-Linear 1', ou simplesmente TNL-1, para a qual os níveis de intensidade mais baixos da imagem original são multiplicados por fatores de escala mais baixos que os aplicados aos níveis mais elevados. As Figuras 3 e 4 ilustram os resultados da aplicação de TNL1 sobre as imagens-teste SUSAN e PILLOW.

De forma a tornar a análise de invariância das respostas dos detectores coerente, cada detector recebeu um conjunto fixo para seus respectivos parâmetros de ajuste, o qual foi mantido ao longo de todas as simulações. Para tal, foram utilizados os seguintes valores de parâmetros: (desvio padrão $=0,6$, limiar inferior de histerese $=0,3$, limiar superior de histerese $=0,9)$ para o detector de Canny conforme recomendado em [7], (escala_1 $=1,2$ e escala_2 $=2,8$ ) para o método de Marr-Hildreth conforme recomendado em [7], (limiar =20,0) para o método SUSAN conforme sugerido em [3]. Para o detector de borda proposto, foram utilizadas duas escalas fixas (escala_1 $=2,3$ e escala_2 $=4,8$ ), segundo simulações preliminares conduzidas pelos autores.

A seguir, são mostrados alguns resultados representativos para as simulações realizadas. As Figuras 3 e 4 mostram as imagens-teste PILLOW e SUSAN em suas versões originais e 'modificadas' por TNL-1 e seus respectivos histogramas. A imagem PILLOW corresponde a uma cena natural, e apresenta uma grande região homogênea (o travesseiro), cercada por diferentes níveis de detalhes. Esta imagem contém regiões com muita textura e objetos com formas variadas. A imagem SUSAN é uma imagem sintética, com estruturas 2-D contendo bordas acentuadas e junções com formas geométricas diversas, bem como variações em tons de cinza suaves e bruscas. Tais características nem sempre são encontradas em imagens de cenas não sintéticas. Esta imagem é interessante para a análise do desempenho dos algoritmos em junções, no tocante à sua localização, conectividade $\mathrm{e}$ preservação da forma.

As Figuras 5 a 8 mostram, respectivamente, os mapas de bordas para as imagens PILLOW e SUSAN em suas versões originais e 'modificadas', obtidos pelos métodos de Marr-Hildreth, Canny, SUSAN e pelo método proposto.

Para a imagem PILLOW (Figuras 5 e 6), o médoto de Marr-Hildreth apresentou resultados relativamente invariantes para as versões original e 'modificada'. No entanto, as bordas mostraram-se grossas e com comprometimento da precisão de localização, algumas bordas foram falsamente detectadas ou não detectadas e a conectividade nas junções foi pobre. Para o detector de Canny, os mapas resultantes foram bastante discrepantes, com bastante ruído e um grande número de falsos positivos para a versão original da imagem, enquanto diversas bordas foram perdidas para a versão 'modificada'. O operador SUSAN perdeu diversas bordas para a versão original e praticamente não conseguiu detectar contornos usando o valor sugerido de limiar 'default'. Comparado aos demais algoritmos, o método proposto apresentou diferenças insignificantes para os dois mapas obtidos, com bordas bem localizadas, finas, contínuas, baixo nível de ruído e boa conectividade nas junções.

Para as versões original e 'modificada' da imagem sintética SUSAN (Figuras 7 e 8), o operador de Marr-Hildreth manteve sua característica de invariância de resposta a manipulações de brilho e contraste, às custas de bordas grossas e mal localizadas, e junções distorcidas, deslocadas e mal conectadas. Para o método de Canny, diversos pontos dos contornos das formas geométricas e da rampa em tons de cinza localizada à esquerda não foram detectados em ambas as imagens. $\mathrm{O}$ algoritmo SUSAN exibiu boa invariância de resposta e localização, com erros praticamente desprezíveis na localização e preservação da forma em algumas junções e detecção de falsos positivos no retângulo localizado no canto inferior direito da imagem 'modificada'. $\mathrm{O}$ erro 
mais perceptível foi a perda das transições mais suaves da rampa de luminância à esquerda da versão 'modificada' desta imagem-teste. Novamente o algoritmo proposto manteve-se como o mais invariante dentre os analisados, apresentando como principal problema uma resposta ruidosa no interior do retângulo situado no canto inferior direito da imagem 'modificada'. Também a precisão de localização de bordas manteve-se muito boa, e apenas para o caso de junções mais complexas, formadas pela interseção de três segmentos de reta, o desempenho de localização e preservação da estrutura foi algo inferior ao do operador SUSAN.

De forma geral, os exemplos aqui apresentados repetiram os resultados obtidos nas simulações conduzidas.

Os mapas de borda gerados pelo método de Marr-Hildreth mostraram-se relativamente robustos às manipulações de brilho e contraste das imagens, o que é uma consequência direta deste operador ter sido desenvolvido baseado em cruzamentos por zero das segundas derivadas das intensidades das imagens. Entretanto, as bordas são em geral grossas e com localização imprecisa. Este fato é devido à aproximação do campo vetorial associado às derivadas direcionais por um valor escalar, dado pelo laplaciano da intensidade da imagem, bem como falsas respostas dos cruzamentos por zeros a pontos que não sejam máximos locais, tais como pontos de mínimo e de inflexão da primeira derivada. As áreas da imagem com estrutura mais complexa são particularmente afetadas, pelo efeito cumulativo desta imprecisão analítica com as operações de filtragem.

Os métodos de Canny e SUSAN apresentam bordas finas e com localização precisa, sendo que o detector de Canny é ainda hoje um dos mais importantes algoritmos de referência na área. No entanto, os mapas de borda produzidos por ambos os métodos são particularmente sensíveis a transformações aplicadas sobre a função intensidade das imagens, mostrando-se ruidosos (muitos falsos positivos) em regiões com alta relação de contraste e perdendo contornos verdadeiros (falsos negativos) em áreas de baixo contraste. Esta é uma consequência de suas formulações analíticas, baseadas em última análise em atributos da própria intensidade dos pixels ou das primeiras derivadas desta. Por este motivo, os mapas de borda produzidos variam consideravelmente para diferentes valores de seus parâmetros de ajuste, fazendo com que imagens obtidas com diferentes níveis de brilho e contraste demandem grandes variações nos parâmetros internos para estes detectores de borda. Por ser baseado diretamente na função intensidade da imagem, o método SUSAN é o que apresenta melhor localização e preservação da forma para junções com estruturas mais complexas. Este é reconhecidamente um dos pontos fortes deste detector, fato este contrabalançado por sua susceptibilidade a ruído e contraste local da imagem cujos contornos se deseja obter.

Para o detector proposto neste trabalho, os comentários fundem-se parcialmente às conclusões finais, e serão então desenvolvidos na seção a seguir.

\section{CONCLUSÕES}

Neste trabalho foi proposto um detector de bordas cujos principais motivadores foram um elevado desempenho de detecção e robustez a variações de brilho e contraste das imagens a serem analisadas. Coerentemente com os princípios e objetivos que nortearam seu desenvolvimento, nas simulações realizadas foram obtidas bordas finas, bem localizadas, e com elevada invariância em relação à aplicação de transformações lineares e não lineares às intensidades dos pixels das imagens, para um mesmo conjunto de parâmetros de entrada para o algoritmo (no caso, as escalas dos filtros gaussianos utilizados).
Também graças a verificações adicionais introduzidas sobre os pontos candidatos a bordas, o método levou a um baixo número de falsos positivos e negativos quando comparado a outros dos principais algoritmos da área.

Mesmo áreas das imagens com estruturas mais complexas exibiram bons resultados, apesar das operações de filtragem implícitas no método. Isto pode ser justificado pela maior precisão da modelagem analítica utilizada. No entanto, para áreas com maior grau de textura ou junções com estruturas mais complexas, uma maior localização, conectividade e preservação da estrutura podem ser obtidas por uma seleção mais criteriosa para as escalas dos filtros.

Nas simulações apresentadas, foram utilizadas para o método proposto escalas fixas para os filtros gaussianos, determinadas a partir de resultados preliminares obtidos pelos autores. Desta forma, a implementação de adaptatividade local para as escalas dos filtros poderá levar a um aumento do desempenho final do algoritmo, sendo esta uma sugestão para trabalhos futuros.

\section{AGRADECIMENTOS}

Os autores agradecem ao Instituto Militar de Engenharia e o apoio do Centro Tecnológico do Exército e da FINEP, sob o convênio no. 2645/06 FINEP/FAPEB.

\section{REFERENCIAS}

[1] D. Marr and E. C. Hildreth, "Theory of Edge Detection", Proceedings of the Royal Society of London, vol. B 207, pp. 187-217, 1980.

[2] J. Canny, "A Computational Approach to Edge Detection", IEEE Transactions on Pattern Analysis and Machine Intelligence, vol. 8, no. 6, pp. 679-698, 1986.

[3] S. M. Smith and J. M. Brady, "SUSAN - A New Approach to Low Level Image Processing", International Journal of Computer Vision, vol. 23, no. 1, pp. 45-78, 1997.

[4] M. Basu, "Gaussian-Based Edge-Detection Methods - A Survey”, IEEE Transactions on Systems, Man, and Cybernetics - Part C: Applications and Reviews, vol. 32, no. 3, pp. 252-260, 2002.

[5] D. Ziou and S. Tabbone, "Edge Detection Techniques - An Overview", International Journal of Pattern Recognition and Image Analysis, vol. 8, pp. 537-559, 1998.

[6] http://figment.csee.usf.edu/ sarkar/resume/default.htm [7] M. Heath, S. Sarkar, T. Sanocki, K. Bowyer, "Comparison of Edge Detectors: A Methodology and Initial Study”, IEEE Computer Society Conference on Computer Vision and Pattern Recognition 1996, CVPR'96, 1996.

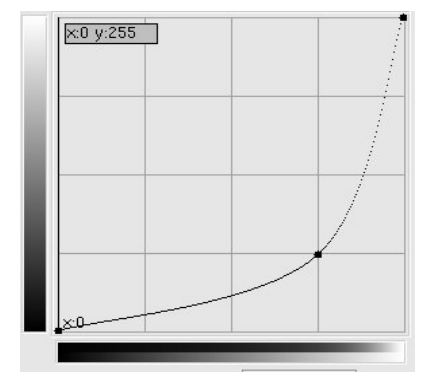

Fig. 2. Transformação não linear TNL-1. 

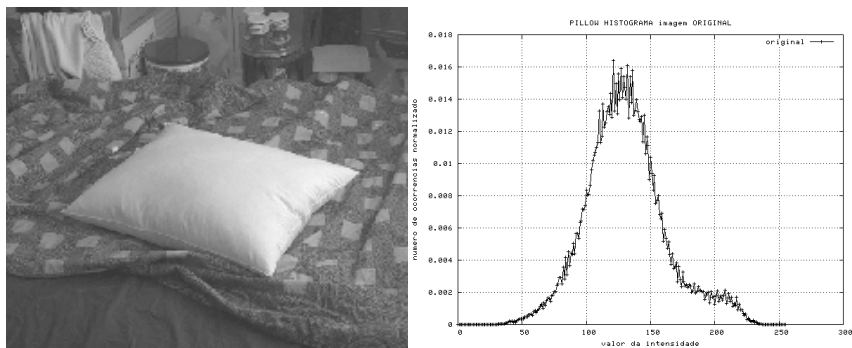

(a)
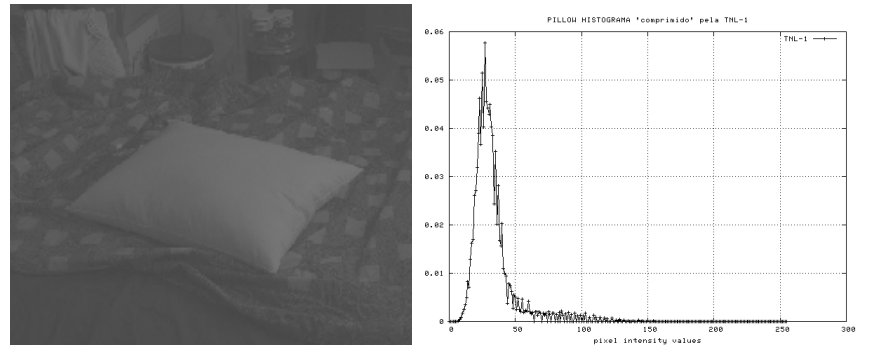

(b)

Fig. 3. Imagem PILLOW e histograma: (a) original; (b) 'modificada' após aplicação de TNL-1.
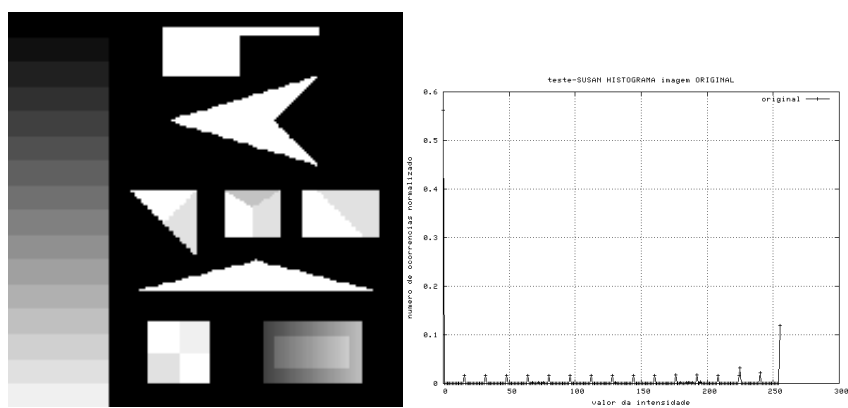

(a)

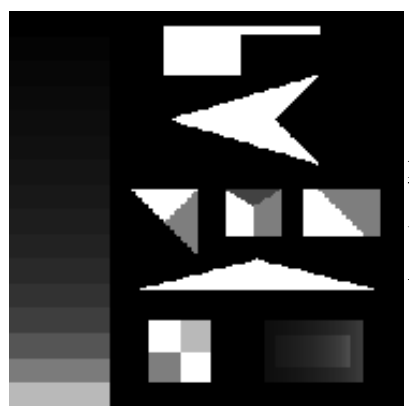

(b)

Fig. 4. Imagem SUSAN e histograma: (a) original; (b) 'modificada' após aplicação de TNL-1.

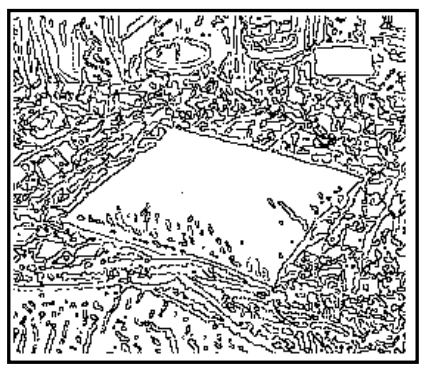

(a)

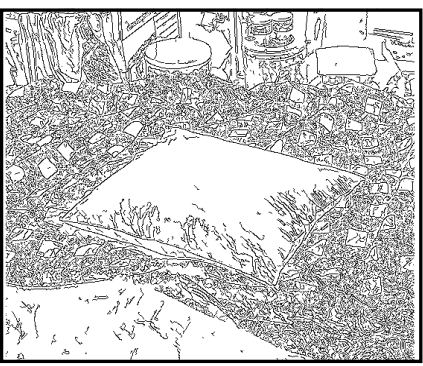

(b)

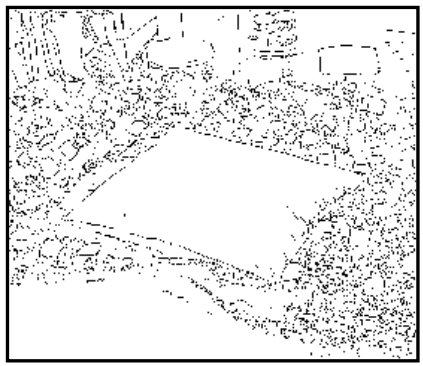

(c)

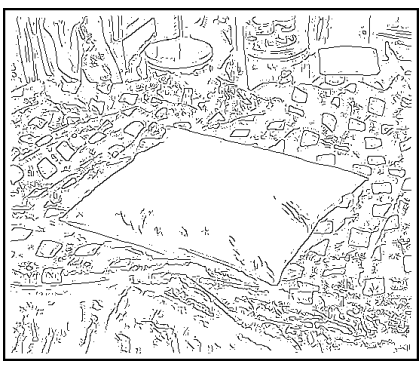

(d)

Fig. 5. Mapas de borda da imagem PILLOW original: (a) Marr-Hildreth; (b) Canny; (c) SUSAN; (d) método proposto.

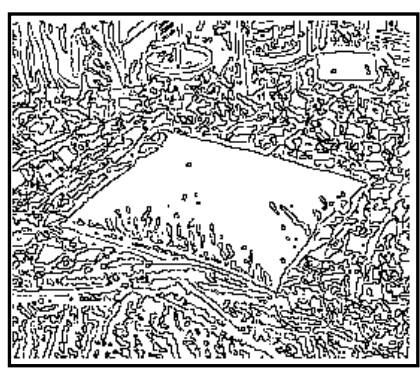

(a)

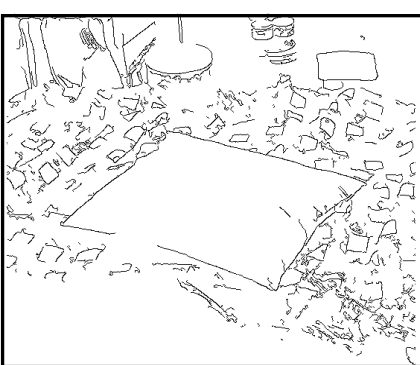

(b)

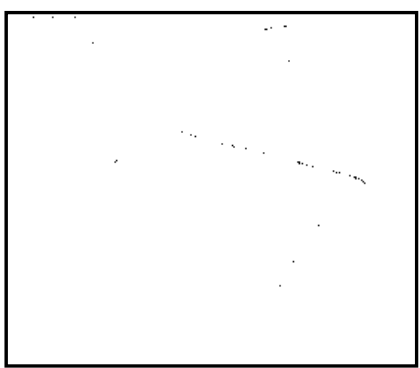

(c)

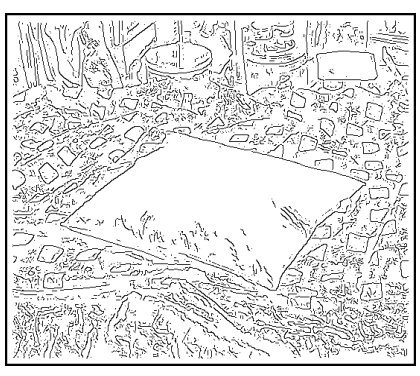

(d)

Fig. 6. Mapas de borda da imagem PILLOW 'modificada': (a) Marr-Hildreth; (b) Canny; (c) SUSAN; (d) método proposto. 


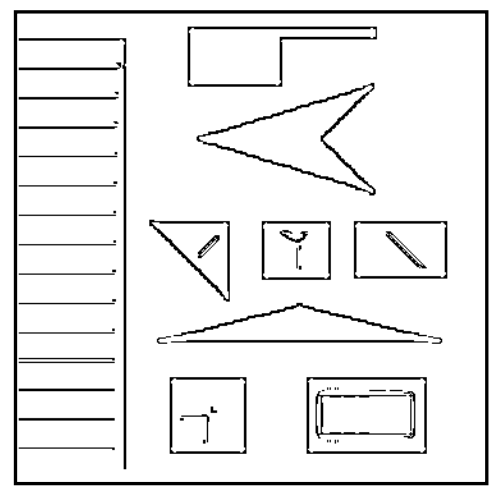

(a)

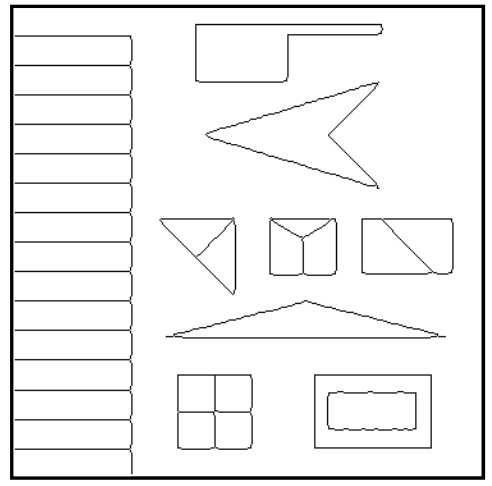

(c)

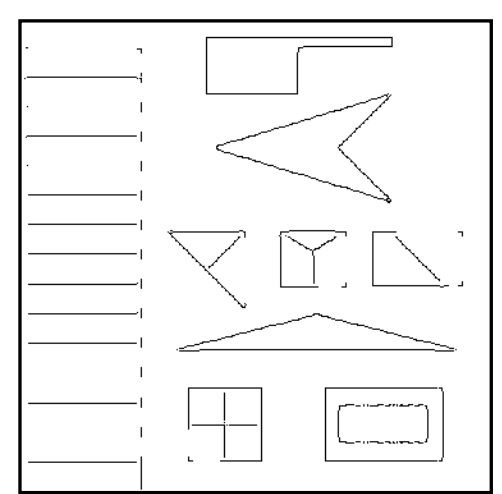

(b)

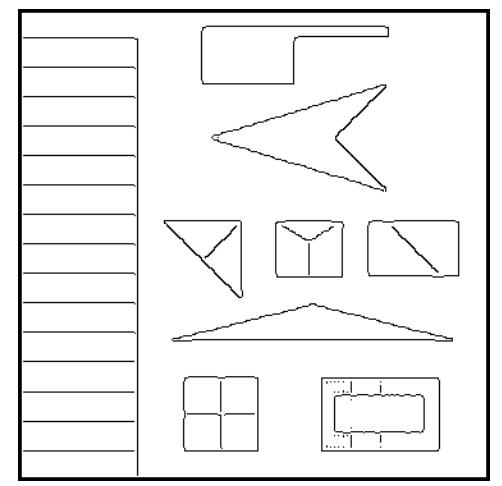

(d)

Fig. 7. Mapas de borda da imagem SUSAN original:(a) Marr-Hildreth; (b) Canny; (c) SUSAN; (d) método proposto.

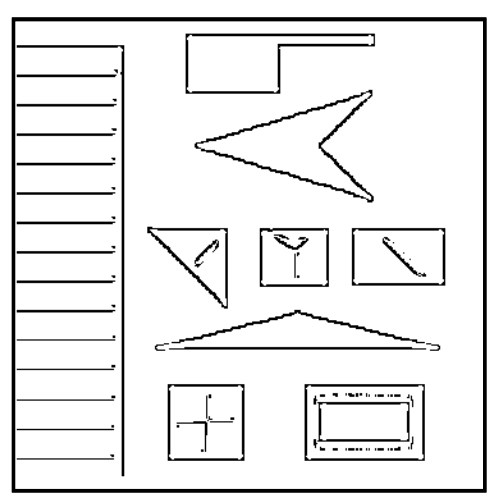

(a)

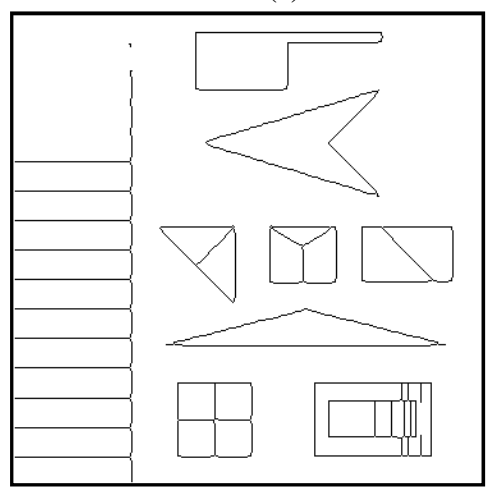

(c)

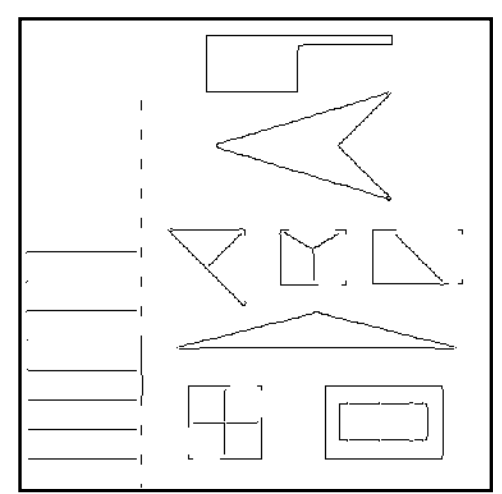

(b)

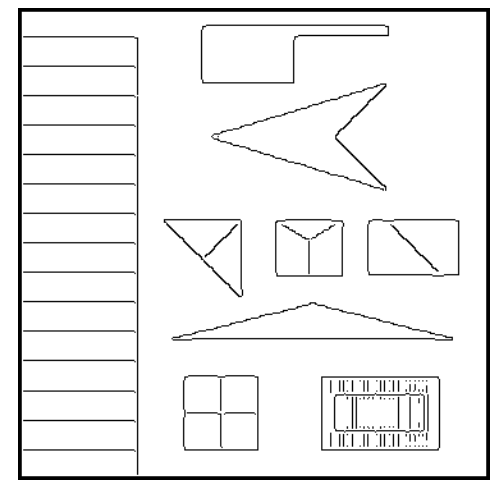

(d)

Fig. 8. Mapas de borda da imagem SUSAN 'modificada': (a) Marr-Hildreth; (b) Canny; (c) SUSAN; (d) método proposto. 